${ }^{1}$ Centro de Investigación CIDCIE, Universidad del Bío-Bío. Chillán, Chile. aphD.

bLicenciada en Educación. ¿Licenciado en Trabajo Social.

Trabajo no recibió financiamiento.

Los autores declaran no tener conflictos de interés.

Recibido el 20 de julio de 2020, aceptado el 19 de mayo de 2021.

Correspondencia a: Dr. Carlos Rodríguez Garcés Centro de Investigación CIDCIE, Universidad del Bío-Bío. Chillán, Chile. carlosro@ubiobio.cl

\section{Cáncer y acción preventiva en Chile: perfilando la abstención a la mamografía y papanicolaou}

\author{
CARLOS RODRÍGUEZ-GARCÉS ${ }^{1, a}$, \\ DENISSE ESPINOSA-VALENZUELA $A^{1, b}$, \\ GERALDO PADILLA-FUENTES ${ }^{1, c}$
}

\section{Abstention from pap smears and mammography among chilean women. Prevalence and causes}

Background: Cancer is a public health problem among women, but preventive actions are effective. Aim: To outline the reasons and factors associated with abstention from pap smears and mammography examinations among women. Material and Methods: Using official databases of the WHO and the Ministry of Social Development of Chile, inferential statistical analyses and modeling of abstention through binomial logistic regression were carried out. Results: Breast and cervical cancer had the highest incidence and mortality among women over 35 years old in Chile, even when there are effective screening methods for their early detection. Abstention is conditioned by personal reasons such as low risk perception. There is a high concomitance of abstention for pap smears and mammography. Conclusions: Health promotion actions should encourage women to undergo preventive actions for breast and cervical cancer.

(Rev Med Chile 2021; 149: 1150-1156)

Key words: Breast Neoplasms; Early Diagnosis of Cancer; Papanicolaou Test; Uterine Cervical Neoplasms.
$\mathrm{E}$ 1 cáncer es una patología compleja, diversa y multicausal y constituye en uno de los mayores desafíos que enfrenta la medicina y la política pública en razón de su alta tasa de incidencia y mortalidad ${ }^{1,2}$. Causas genéticas-hereditarias, junto con factores ambientales y estilos de vida, se asocian a su aparición ${ }^{3}$. Si bien el cáncer en mujeres respecto de hombres registra una menor incidencia y mortalidad ${ }^{4}$, en ellas resultan particularmente alarmante en atención a la mortalidad que alcanzan tipologías específicas como el cáncer mamario (C50) y ginecológico (C51-C56 $)^{5-9}$.

En mortalidad, el cáncer de mamas en mujeres es la décima causa de muerte a nivel general y la primera de etiología oncológica ${ }^{10,11}$; mientras, el cáncer cervicouterino ocupa el lugar 27 entre todas las causas de muerte y el 5 entre las oncológicas.

Factores como el envejecimiento poblacional y la consistente pérdida de capacidad del cuerpo para combatir neoplasias ${ }^{12}$, unido a la falta de actitud preventiva, han hecho que la mortalidad por estas patologías avance significativamente $\mathrm{e}^{13,14}$.

La detección en fase avanzada, ausencia de diagnóstico y debilidad en los tratamientos, son problemas recurrentes, particularmente acentuados en países más pobres y entre población de menor escolaridad ${ }^{4}$. La desigualdad económica y educativa inciden significativamente en la mortalidad por cáncer debido al segregado acceso y deficiente calidad de los servicios médicos ${ }^{15}$, 
asimismo el temor, la desinformación y el pudor inhiben la realización del examen preventivo ${ }^{16-21}$.

La acción preventiva de autocuidado cobra relevancia toda vez que la cancerogénesis es un proceso lento y generalmente asintomático ${ }^{22}$. Las mamografías para la pesquisa de anormalidades a veces imperceptible a la autoexploración, así como el tamizaje de papanicolaou para la detección de la presencia del virus de papiloma humano (VPH), han demostrado alta eficacia y efectividad en la detección temprana ${ }^{23,24}$.

En Chile, tanto el examen de papanicolaou como la mamografía forman parte del Programa Nacional del Cáncer, iniciativa de carácter público, acceso gratuito para población en riesgo. No obstante, se constatan importantes brechas en sus tasas de cobertura, a lo que se suma una alta abstención femenina a repetir screening alterados ${ }^{25}$. Ello pone en evidencia la necesidad de complementar las acciones de prevención y detección temprana con programas de educación y concientización respecto al autocuidado. $\mathrm{Al}$ ser esta una decisión personal, está fuertemente modelada por la motivación que regula las conductas preventivas o de autocuidado ${ }^{26,27,21}$.

Considerando la relevancia del cáncer como fenómeno epidemiológico para la política pública, es objetivo de este artículo exponer las tasas de prevalencia, incidencia y mortalidad que tienen en Chile los cánceres que afectan a mujeres, en particular mamario y cervicouterino. Debido a la importancia de la acción preventiva, se hace hincapié en perfilar los niveles de abstención a los exámenes de mamografía y papanicolaou $y$, mediante modelos de regresión logística, se configuran aquellos factores que condicionan tal abstención.

\section{Material y Método}

Se realizó un análisis estadístico en dos etapas según los objetivos y naturaleza de las BBDD Global Cancer Observatory (GCO) y Encuesta de Caracterización Socioeconómica Nacional (CASEN) del año 2017, combinando análisis descriptivos e inferenciales en el contexto bivariante y multivariante. En la primera etapa, se establece prevalencia, incidencia y mortalidad de las neoplasias malignas desarrolladas en mujeres de 35 años y más (C50-C54; C56). En la siguiente fase, se cuantifican los niveles de abstención a la acción preventiva del papanicolaou y mamografía, los motivos y predictores de riesgo mediante modelos de regresión logística binomial (BR).

Con la base de datos reportados por GCO de la Agencia Internacional de Investigación del Cáncer de la Organización Mundial de la Salud (OMS) al año 2020, se construyeron un conjunto de índices para dimensionar la situación de los cánceres de mama y ginecológicos en Chile:

- Prevalencia: número de mujeres de 35 años o más que han sido diagnosticadas, están en tratamiento o se consideran curadas de cáncer, dentro de un plazo específico de 5 años. Se expresa en número de casos y proporciones por cada 100.000 habitantes de la población en riesgo.

- Incidencia: cociente del número de casos nuevos de cáncer que surgen en la población de mujeres mayores de 34 años durante un año.

- Mortalidad: índice de mujeres mayores de 35 o más años muertas por cáncer durante un año.

Los índices de incidencia y mortalidad son expresados como:

- $\quad N^{\circ}$ casos: número o valor absoluto de nuevos casos o muertes registradas por tipo de cáncer y población específica.

- Tasa bruta: cociente del número de nuevos casos detectados o muertos por cáncer observados durante un año, dividido por el total de mujeres de 35 años o más, expresado por cada 100.000 mujeres en riesgo.

- Tasa estandarizada por edad (ASR): a fin de controlar la influencia que tiene la edad en el riego de padecer o morir de un cáncer y hacer las medidas comparables entre poblaciones diferentes, las tasas son estimadas y ponderadas sobre la base de una estructura etaria estándar para la población mundial.

- Riesgo: probabilidad estimada que tienen las mujeres de desarrollar o morir por algún tipo de cáncer durante un tiempo determinado.

- Predominio: estimación del peso o magnitud que tiene un tipo de cáncer específico respecto del total de cánceres asociados a la población de estudio, en su incidencia o mortalidad. Índice expresado como porcentaje.

En un segundo apartado, se modeló la abstinencia a la acción preventiva de mamografía y examen de papanicolaou con datos CASEN en su 
versión 2017, estableciéndose prevalencias y motivos de la abstención segmentados por capital educativo. La literatura reporta como relevante esta variable frente a la acción preventiva. Estos análisis fueron complementados con sendos modelos de regresión logística binaria (BR), consignando para este efecto los predictores teóricamente más significativos, recodificando algunos recodificados en lenguaje binario según grupo de riesgo: escolaridad (mediana y alta escolaridad $=0$; baja escolaridad $=1)$, edad ( $<60$ años $=0 ;>60$ años $=1)$, zona ( urbano $=0$; rural $=1)$, pobreza $(60 \%$ más rico $=0 ; 40 \%$ más pobre $=1)$, tenencia de pareja (no tiene $=0$; tiene $=1), P A P($ no se abstiene $=0$; se abstiene $=1$ ), mamografía (no se abstiene $=0$; se abstiene $=1$ ). Conforme se modele la abstención al papanicolaou o mamografía, estas variables fueron también utilizadas como predictores a fin de evaluar su acción concomitante.

\section{Resultados}

Según reporta el GCO, al año 2018 se produjeron a nivel mundial un total de 9.555.027 muertes por cáncer (IC ,95 [9.345.400-9.769.350]), de las cuales $43,6 \%$ fueron mujeres. En Chile, los decesos totales fueron 28.443 (IC ,95 [28.044-2.848]) y un $47,3 \%$ correspondió a mujeres (n: 13.463; IC ,95 [13.183-13.749]) (Tabla 1).

Del total de mujeres mayores de 34 años muertas en Chile por alguna patología oncológica $(\mathrm{n}=13.214), 1$ de cada 4 decesos $(24,1 \%)$ se asocia a una neoplasia maligna localizada en la zona mamaria (C50; 12,6\%) o zona genital como: cuello uterino (C53; 5,3\%), ovárico (C56; 3,5\%), cuerpo uterino (C54; 2,2\%), vulva (C51; $0,4 \%)$ o vagina (C52; $0,1 \%)$.

A nivel nacional, del total de mujeres muertas por cáncer, un $17,9 \%$ lo son a causa del tipo mamario o uterino. De los 3.190 decesos registrados en las tipologías analizadas, estos cánceres concentran el 73,9\%.

En los últimos 5 años se registra en el país un total de 19.702 casos diagnosticados de cáncer Mamario. La incidencia es de 109,8 casos nuevos por cada 100.000 habitantes de población en riesgo y con una tasa ajustada por edad ASR de 101,3. En ambos casos, levemente inferior a la exhibida en el contexto latinoamericano. A nivel global, esto es, considerando todos los cánceres, 1 de cada 5 (20,9\%) nuevos casos diagnosticados son patologías del tipo mamario. Por su parte, la mortalidad registra una tasa bruta de 35,5 y una ASR de 28,7.

Tabla 1. Descriptores epidemiológicos por tipos de Ca, mujeres 35 años y más

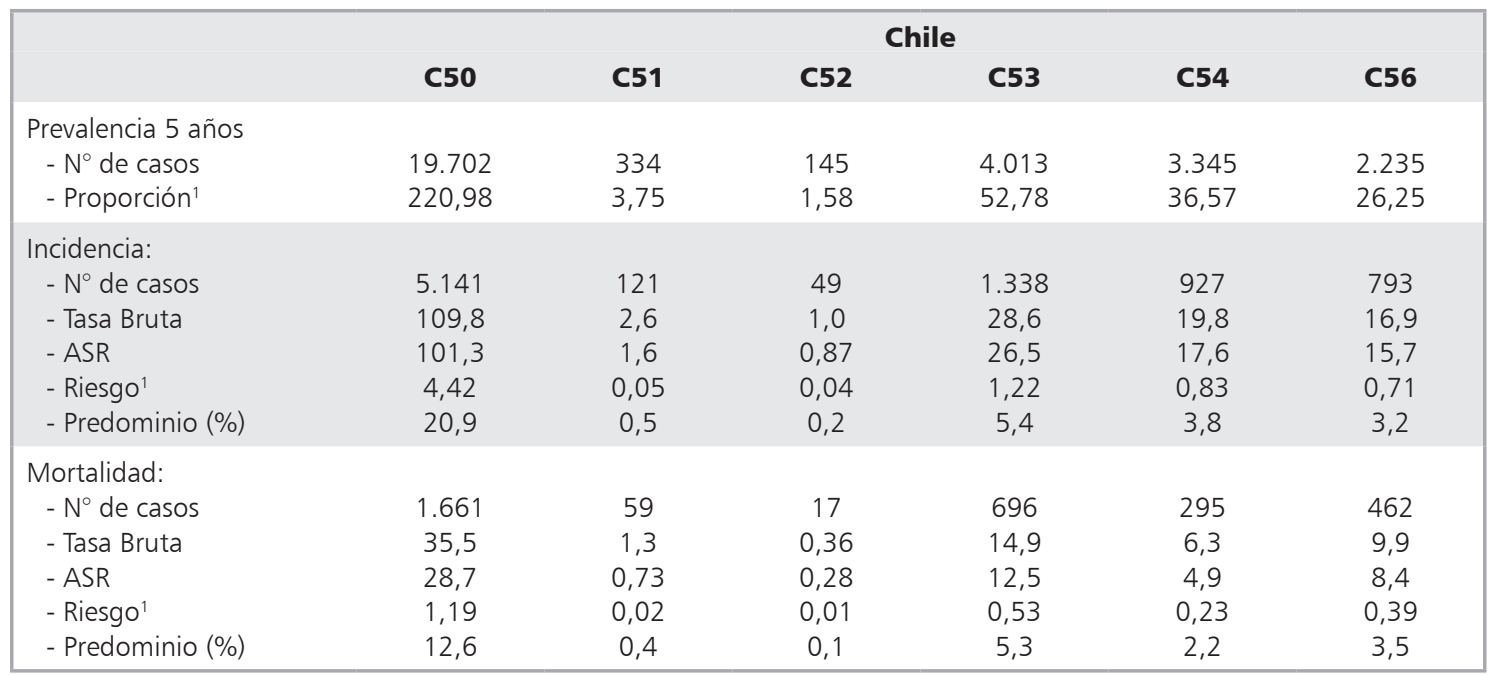

Nota: 1 = El índice se calcula sobre la base del total de mujeres, incluye todas las edades, C50= Ca de mamas; C51=Ca de vulva; $\mathrm{C} 52=$ Ca de vagina; $\mathrm{C} 53=$ Ca Cuello uterino; $\mathrm{C54}=$ Ca Cuerpo uterino; C56= Ca ovárico, Fuente: Elaboración propia sobre la base de Global Cancer Observatory of International Agency for Research by World Health Organization (2018). 
El 12,6\% de las mujeres mayores de 34 años fallecidas es debido a esta patología oncológica.

En mujeres, el riesgo de morir por cáncer mamario es 5,2 veces superior al riesgo de deceso por cáncer en el cuerpo uterino; 3,1 veces mayor respecto al cáncer ovárico y 2,3 veces en comparación al cáncer uterino.

Los otros cánceres asociados a mujeres tienen una significativa menor prevalencia, incidencia y mortalidad. En estas neoplasias encontramos una mayor incidencia de carcinomas vinculados al cuello uterino (n: 1.338; ASR: 26,5; riesgo: 1,22), cuerpo uterino (n: 927; ASR: 17,6; riesgo: 0,83) y ovárico (n: 793; ASR: 15,7; riesgo: 0,71).

En términos de prevalencia, incidencia y mortalidad, el cáncer al cuello uterino se ubica en el segundo lugar después del cáncer mamario, con significativas diferencias en los distintos índices respecto de los otros cánceres analizados.

Si bien el riesgo de incidencia de cáncer de cuerpo uterino es mayor que el ovárico $(0,81$ versus 0,71 ), al momento de analizar las tasas de mortalidad, el riesgo de fallecer por este último es 1,7 veces mayor que el registrado por el primero (Tabla 2).

Dada la importancia epidemiológica de las patologías cancerígenas mamarias y cérvico uterinas, se indagó respecto de la acción preventiva considerando cada población en riesgo. La mamografía registra $31,5 \%$ de abstención y el papanicolaou $22,9 \%$, con diferencias estadísticamente significativas por nivel de escolaridad, tanto en el primer $\left[\chi^{2}=270,625 ; \mathrm{p}<0,00\right]$ como segundo examen $\left[\chi^{2}=53,635 ; \mathrm{p}<0,00\right]$. La menor escolaridad se constituye en un factor de riesgo para la imple- mentación del autocuidado, aunque los niveles de abstención son igualmente preocupantes entre mujeres más educadas.

Los motivos de abstención registran una prevalencia jerárquica similar con independencia del tipo de examen. El 49,4\% no considera necesario realizarse una mamografía, y 46,6\% esgrime idéntico motivo para abstenerse de papanicolaou, mientras que el desconocimiento registra prevalencias de $16,8 \%$ y $21,6 \%$ respectivamente. Las barreras institucionales o falta de recursos, tienen una presencia marginal $(9,7 \%$ y $7,1 \%$ según tipología de examen).

Contrastando por nivel educativo, los motivos de abstención siguen el mismo patrón que la prevalencia general, pero con leves diferencias. En la mamografía, el motivo de abstención por no considerarlo necesario alcanza 51,6\% entre mujeres con 8 o menos años de escolaridad, con una diferencia de 4,1 puntos porcentuales sobre el grupo de comparación.

Las barreras institucionales tienen una presencia marginal, sin embargo, se evidencia un mayor peso comparativo entre las mujeres con menor escolaridad (10\%). Similar tendencia se observa al comparar los motivos para abstenerse al papanicolaou (Tabla 3 ).

Al momento de configurar los factores de riesgo, los modelos de regresión observan concomitancia entre abstención a examen de mamografía y de papanicolaou, de modo que no realizarse uno de ellos deriva en una alta probabilidad de abstención al otro. El riesgo de abstención de la mamografía se eleva 22 veces cuando no se ha realizado el papanicolaou. Controlando por edad

Tabla 2. Abstención a la acción preventiva de mamografía y papanicolaou según años de escolaridad

\begin{tabular}{|lcccccc|}
\hline & \multicolumn{3}{c}{ Mamografía $(\mathbf{n}=\mathbf{3 0 . 5 9 5})$} & \multicolumn{3}{c|}{ Papanicolaou (n= 55.133) } \\
& $<\mathbf{8}$ años & $\mathbf{1 3}$ y más & Total & $<\mathbf{8}$ años & $\mathbf{1 3}$ y más & Total \\
Déficit de acción preventiva (\%) & 36,0 & 24,7 & 31,5 & 24,9 & 23,1 & 22,9 \\
Razones de abstención & & & & & & \\
- Desconocimiento & 16,3 & 16,9 & 16,8 & 20,1 & 21,2 & 21,6 \\
- Miedo & 10,8 & 13,5 & 11,9 & 14,2 & 9,2 & 12,1 \\
- No lo considera necesario & 51,6 & 47,5 & 49,4 & 45,4 & 50,1 & 46,6 \\
- Barreras institucionales o recursos & 10,0 & 7,3 & 9,7 & 8,1 & 5,7 & 7,1 \\
- Otra & 11,2 & 14,8 & 12,1 & 12,2 & 13,8 & 12,5 \\
\hline
\end{tabular}

Nota: índices de abstención calculados para población objetivo recomendada por guía clínica del Ministerio de Salud de Chile; para la mamografía se consideraron mujeres entre 50 y 74 años, y para papanicolaou mujeres entre 25 y 64 años. Fuente: Elaboración propia con datos CASEN (2017). 
Tabla 3. Modelo RLB para riesgo de abstención de examen de mamografía y papanicolaou

\begin{tabular}{|c|c|c|c|c|}
\hline & \multicolumn{2}{|c|}{ Mamografía } & \multicolumn{2}{|c|}{ Papanicolaou } \\
\hline & Wald & $\operatorname{Exp}(B)$ & Wald & $\operatorname{Exp}(B)$ \\
\hline Escolaridad (años) & 50,557 & $0,970 * *$ & 10,201 & $0,987 * *$ \\
\hline Edad (años) & 1,218 & 1,003 & 1255,333 & $1,068^{* *}$ \\
\hline Zona Rural & 0,582 & 0,968 & 15,964 & $0,856^{* *}$ \\
\hline Decil de ingresos & 87,980 & $0,940 * *$ & 12,430 & $1,021 * *$ \\
\hline Tenencia de pareja & 70,051 & $0,759 * *$ & 116,295 & $0,729 * *$ \\
\hline Abstención PAP ${ }^{1}$ & 8515,572 & $22,102 * *$ & -- & -- \\
\hline Abstención Mam¹ & -- & -- & 7979,331 & $18,219 * *$ \\
\hline Constante & 83,483 & $0,231 * *$ & 2263,133 & $0,003 * *$ \\
\hline Correctamente clasificados (\%) & \multicolumn{2}{|c|}{84,1} & \multicolumn{2}{|c|}{83,4} \\
\hline Log verosimilitud -2 & \multicolumn{2}{|c|}{24995,096} & \multicolumn{2}{|c|}{31224,673} \\
\hline R2 de Cox y Snell & \multicolumn{2}{|c|}{0,339} & \multicolumn{2}{|c|}{0,242} \\
\hline R2 de Nagelkerke & \multicolumn{2}{|c|}{0,476} & \multicolumn{2}{|c|}{0,372} \\
\hline
\end{tabular}

Nota: 1 = variable modelada utilizada como regresor en uno de los modelos; *significativo al ,95; **significativo al ,99; índices de abstención calculados para población objetivo recomendada por guía clínica del Ministerio de Salud de Chile; para la mamografía se consideraron mujeres entre 50 y 74 años, y para papanicolaou mujeres entre 25 y 64 años; PAP = papanicolaou; Mam = mamografía. Elaboración propia con datos CASEN (2017).

recomendada, del total de 9.673 mujeres que no se realizaron la mamografía, un $75,6 \%$ se abstiene de realizarse el papanicolaou. A su vez, de las 12.688 mujeres que no se realizaron papanicolaou, $77,8 \%$ se abstiene de mamografía.

En lo que a mamografía refiere, el riesgo de abstención disminuye conforme mejoran los deciles ingresos $[\operatorname{Exp}(\beta)=0,940]$ y escolaridad $[\operatorname{Exp}$ $(\beta)=0,970$, al igual que cuando se tiene pareja $[\operatorname{Exp}(\beta)=0,759]$.

Por su parte, en el modelamiento de abstención a papanicolaou, la edad $[\operatorname{Exp}(\beta)=1,068]$ y el decil de ingresos $[\operatorname{Exp}(\beta)=1,021]$ aumentan progresivamente el riesgo, mientras que la escolaridad $[\operatorname{Exp}(\beta)=0,987]$, residir en territorio rural $[\operatorname{Exp}(\beta)=0,856]$ y tener pareja $[\operatorname{Exp}(\beta)=0,729]$ constituyen factores que estimulan el autocuidado.

\section{Discusión}

Entre el cáncer mamario, de cuerpo uterino y ovárico, el primero es la patología oncológica de mayor prevalencia, incidencia y principal causa de muerte en mujeres de 35 y más años en Chile, lo cual justifica su preocupación como política pública. Aunque sus tasas de mortalidad son menores que el resto de Latinoamérica, aún son superiores en comparación con regiones desarrolladas como Norteamérica y Europa ${ }^{28,29}$.

Las desigualdades en el diagnóstico y tratamiento oportuno, además de una actitud despreocupada hacia el control preventivo, explicarían sus elevadas tasas de mortalidad y concentración en edades inferiores a la exhibida por países desarrollados ${ }^{11,30,31}$.

Barreras institucionales coexisten con actitudes de riesgo que potencian la abstención frente la acción preventiva. En Chile, los exámenes de mamografía y papanicolaou son ambulatorios y de acceso gratuito para determinada población objetivo, existiendo un gran conglomerado que no se los realiza. Para ambos perfiles de abstención, predominan los motivos de carácter personal referidos a la utilidad, pertinencia y eficacia percibida, con una prevalencia superior que las barreras de acceso o déficit informacionales.

La prevención es una decisión de carácter personal vinculada a creencias y perfiles actitudinales que determinan conductas de riesgo. Investigacio- 
nes reportan que la autorresponsabilidad y predisposición desfavorable hacia estos exámenes suelen ser más relevantes que restricciones institucionales de acceso, distancia y oportunidad o sofisticados mecanismos de detección precoz ${ }^{26,32-36}$. Esto revela la necesidad de promoción socioeducativa, complementaria con la orientación médica, para sensibilizar a la población objetivo ${ }^{31,37}$.

En el modelamiento de la abstención a la acción preventiva, políticas de acceso focalizado de servicios públicos, así como diferenciados niveles de asequibilidad al examen, determinan la condición de riesgo o protección que factores socioculturales como la pobreza, escolaridad, zona de residencia o incluso la edad presentan.

Entre los factores de riesgo analizados, destaca la acción concomitante de abstención de ambos exámenes. La probabilidad de abstenerse en uno de ellos se eleva exponencialmente cuando no se realiza el otro. Ello devela que la prevención es una actitud sistemática y voluntaria para conservar la salud y prevenir enfermedades; autocuidado que constituye un estilo de vida.

En consecuencia, una política sanitaria de acción preventiva debería focalizar también su atención a la promoción de hábitos de vida saludable y conductas de autocuidado que moderen reticencias y modifiquen perfiles actitudinales de riesgo a la mamografía y papanicolaou.

\section{Referencias}

1. Marañón T, Mastrapa K, Flores Y, Vaillant L, Landazuri S. Prevención y control del cáncer de cuello uterino. Correo Científico y Médico 2017; 21 (1): 187-203.

2. OMS. Informe sobre la situación mundial de las enfermedades no transmisibles. Organización Mundial de la Salud 2011.

3. Ramírez K, Acevedo F, Herrera ME, Ibáñez C, Sánchez C. Actividad física y cáncer de mama: un tratamiento dirigido. Rev Med Chile 2017; 145 (1): 75-84.

4. OMS. Cáncer, datos y cifras. Organización Mundial de la Salud 2018.

5. Moran R, Quintero M. Antecedentes sobre el cáncer cervicouterino en México e importancia de la educación sexual en la prevención temprana en jóvenes y población rural. Medwave [revista en Internet] 2012; $12(7)$.

6. Plummer M, de Martel C, Vignat J, Ferlay J, Bray F, Franceschi S. Global burden of cancers attributable to infections in 2012: a synthetic analysis. Lancet Glob Health. 2016; 4 (9): 609-16.

7. Naranjo L, Mesa Z, Pérez G, Amechea G. Lesiones cervicouterinas en jóvenes fuera de program. Revista Finlay 2017; 7 (3): 207-12.

8. Bray F, Soerjomataram I, Siegel R, Torre L, Jemal A. Global cancer statistics 2018: GLOBOCAN estimates of incidence and mortality worldwide for 36 cancers in 185 countries. ACS Journals 2018; 64 (6): 394-424.

9. Siegel R, Miller K, Jemal A. Cancer Statistics 2018. CA Cancer J Clin. 2018; 68 (1): 7-30. doi:10.3322/ caac. 21442 .

10. Aguilera X, González C, Matute I, Nájera M, Olea A. Serie de salud poblacional: Las enfermedades no transmisibles en Chile. Aspectos epidemiológicos y de salud pública. Centro de Epidemiología y Políticas Públicas en Salud, Universidad del Desarrollo 2016.

11. Icaza G, Núñez L, Bugueño H. Descripción epidemiológica de la mortalidad por cáncer de mama en mujeres en Chile. Rev Med Chile 2017; 145 (1): 106-14.

12. Lenihan D, Cardinale D. Late cardiac effects of cancer treatment. Journal of Clinical Oncology 2012; 30 (30): 3657-64.

13. Serra I, Martínez R, Reyes G, Sierra P, Aguayo C. Envejecimiento y alta prevalencia de estadios avanzados determinan la creciente mortalidad por cáncer mamario en Chile. Revista Chilena de Cirugía 2012; 64 (2): 137 46.

14. Irarrázaval E, Kleinman P, Silva F, Fernández L, Torres C, Fritis M, et al. Quality of life in Chilean breast cancer survivors. Rev Med Chile 2016; 144 (12): 567-76.

15. Ferreccio C, Barriga M, Lagos M, Ibáñez C, Poggi H, González F, et al. Screening trial of human papillomavirus for early detection of cervical cancer in Santiago, Chile. International Journal of Cancer 2013; 133 (2): 916-23.

16. Nigenda G, Caballero M, Robledo L. Barreras de acceso al diagnóstico temprano del cáncer de mama en el Distrito Federal y en Oaxaca. Salud pública de México 2009; 51: 254-62.

17. Musre O. Cáncer de Mama. Epidemiología y Factores de Riesgo. Cuadernos Médico Sociales 2007; 47 (1): 18-30. Obtenido de http://cms.colegiomedico.cl/Magazine/2007/47/1/47_1_4.pdf

18. De Charry L, Carrasquilla G, Roca S. Equidad en la detección del cáncer de seno en Colombia. Revista de Salud Públic 2008; 10 (4): 571-82. Obtenido de http:// www.scielo.org. co/pdf/rsap/v10n4/v10n4a07.pdf

19. Urrutia M, Poupin L, Concha X, Viñales D, Iglesias C, Reyes V. ¿Por qué las mujeres no se toman el Papanicolau?: Barreras percibidas por un grupo de mujeres 
ingresadas al programa de cáncer cervicouterino AUGE. Revista chilena de obstetricia y ginecología 2008; 73 (2): 98-10. Obtenido de https://scielo.conicyt.cl/scielo. php?pid=S071775262008000200005\&script=sci_arttext

20. Peralta M. Cáncer de mama: estrategias de prevención y vigilancia según nivel de riesgo. Revista Médica Clínica Las Condes 2011; 22 (4): 436-43.

21. Desantis C, Ma J, Bryan L, Jemal A. Breast Cancer Statistics, 2013. A Cancer Journal for Clinicians 2014; 64 (1): 52-62.

22. Rodríguez C, Padilla G. Cáncer de cérvix y autorresponsabilidad: perfilando el riesgo de abstención al. Horizonte Médico 2018; 18 (1): 13-22.

23. Desantis C, Ma J, Bryan L, Jemal A. Breast Cancer Statistics, 2013. A Cancer Journal for Clinicians 2014; 64 (1): 52-62.

24. Uchida M. Mamografía de screening y realidad chilena. Revista chilena de radiología 2008; 14 (3): 130-4. Obtenido de https://scielo.conicyt.cl/scielo.php?pi$\mathrm{d}=$ S0717-93082008000300005\&script=sci_arttext

25. López L, Suárez L, Torres L. Detección del cáncer de mama en México: síntesis de los resultados de la Encuesta Nacional de Salud Reproductiva. Salud pública de México 2009; 51 (2): 345-9.

26. López F, Lorenzi D, Poblete O. Follow-up of women with inadequate Pap smears: a prospective cohort study. Sao Paulo Medical Journa 2015; 133 (1): 20-7.

27. Ponce M. La prevención del cáncer de cuello de útero y de mama en servicios de salud y organizaciones no gubernamentales de la ciudad Autónoma de Buenos Aires. Salud Colectiva 2013; 9 (2): 215-33. Obtenido de https://www.redalyc.org/pdf/731/7312941500.

28. Fica A. Prevención del cáncer cérvico-uterino en Chile: mucha vacuna y poco Papanicolau. Rev Chilena Infectol 2014; 31 (2): 196-203.

29. Allemani C, Weir H, Carreira H, Harewood R, Spika D, Wang X, et al. Global surveillance of cancer survival 1995-2009: analysis of individual data for 25676887 patients from 279 population-based registries in 67 countries (CONCORD-2). The Lancet 2015; 385 (9972): 977-1010.

30. Castillo C, Cabrera E, Derio L, Gaete F, Cavada, G. Impact of the Chilean Explicit Guaranties Health System (GES) on breast cancer treatment. Rev Med Chile 2017; 145 (12): 1507-13. Obtenido de https://dx.doi. org/10.4067/s0034-98872017001201507.

31. Coleman M. Supervivencia del cáncer: la vigilancia mundial estimulará la política de salud y mejorará la equidad. The Lancet 2014; 383: 564-73.

32. MINSAL. Guía Clínica AUGE: Examen medicina preventiva. Santiago de Chile 2013. Obtenido de https:// www.minsal.cl/examen-medicina-preventiva/

33. Rodríguez C, Padilla G. Cáncer mamario y cervicouterino: el problema del absentismo femenino en la acción preventiva. Universidad y Salud 2019; 22 (1): 41-51.

34. Torres G, Ortega C, Ángeles A, Villalobos A, Salmerón J, Lazcano E, et al. Patrones de utilización de programas de prevención y diagnóstico temprano de cáncer en la mujer. Revista de Salud Pública, México 2013; 55 (2): 241-8.

35. Serra I. Cáncer de mama en Chile: pasado, presente y futuro. Revista Chilena de Cirugía 2011; 63 (6): 553-6. Obtenido de http://dx.doi.org/10.4067/S071840262011000600001.

36. Picón M. Conocimientos, percepciones, y prácticas de mujeres jóvenes acerca del papiloma virus humano. Independent Study Project (ISP) Collection 2009; 732: 5-33. Obtenido de https://digitalcollections.sit.edu/ isp_collection/732.

37. Wiesner C. Determinantes psicológicos, clínicos y sociales del diagnóstico temprano del cáncer de mama en Bogotá, Colombia. Revista Colombiana de Cancerología 2007; 11 (1): 13-22. Obtenido de http://www.imbiomed. com/1/1/articulos.php?method=showDetail\&i.

38. Urrutia MT. Creencias sobre Papanicolaou y cáncer cérvicouterino en un grupo de mujeres chilenas. Rev Chil Obstetricia y ginecología 2012; 77 (1): 3-10. Obtenido de http://dx.doi.org/10.4067/S0717-75262012000100002. 